\title{
COVID 19 Pandemic, Mechanism of Pathogenesis, Preventions and Possible Cures to Save Humanity: A Study
}

\author{
Samanwita Banerjee, Jainendra Gupta, Anil Kanaujia \\ Ayurvet Research Foundation, Chidana, Haryana, India
}

Received: 06/05/2020

Accepted: 03/06/2020

Published: 20/06/2020

\begin{abstract}
The name "coronavirus" is derived from Latin corona, meaning "crown" or "wreath due to the characteristic appearance of virions , which have a fringe of large, bulbous surface projections creating an image reminiscent of a crown or of a solar corona. Human coronaviruses were discovered in the 1960s. The third novel coronavirus to emerge in this century is called SARS-CoV-2. It caused coronavirus disease 2019 (COVID-19), which emerged from China in December 2019 and was declared a global pandemic by the World Health Organization on March 11, 2020. The virus enters into the host body through the mouth, nose and eye. From there, the virus makes its way down into the air sacs inside the lungs, known as alveoli. Once in the alveoli, the envelope spike glycoprotein present in the virus binds to its cellular receptor, angiotensin-converting enzyme 2 (ACE2) for SARS-CoV-2. There are two commonly used nucleic acid detection technologies forSARS-CoV-2 are real-time quantitative polymerase chain reaction (RT-qPCR) and high-throughput sequencing. With no sure shot vaccine/ drug in sight till late 2021, medical practioners are using existing therapies and repurposing drugs to save lives. Prevention is the only way to stay safe and healthy. Immunity plays an important role in this disease, so it important have nutritious and healthy food to boost the immunity.
\end{abstract}

Keywords: COVID 19, SARS-CoV-2, Vaccine, ACE2, RT-q PCR

\section{Introduction}

Coronaviruses belongs to the family Coronaviridae, order Nidovirales, which cause diseases in mammals and birds (1-3). The family is further divided into Torovirinae and Coronavirinae sub families. The sub family Coronavirinae is further sub categorized into alpha-, beta-, gamma- and deltaCOVs (2-5). In humans, coronaviruses cause respiratory tract infections that can range from mild to lethal. Among the group there are few lethal varieties which cause SARS, MERS, and COVID-19. The symptom varies with different species such as in chickens, they cause an upper respiratory tract disease, while in cows and pigs they cause diarrhea. It was first discovered in the 1930s when an acute respiratory infection of domesticated chickens was shown to be caused by infectious bronchitis virus (IBV) (3). Human coronaviruses were discovered in the 1960s $(3,6)$. Four of the seven known coronaviruses that sicken people cause only mild to moderate disease. Three can cause more serious, even fatal, disease. SARS coronavirus (SARS-CoV) emerged in November 2002 and caused severe acute respiratory syndrome (SARS). That virus disappeared by 2004. Middle East respiratory syndrome (MERS) is caused by the MERS coronavirus (MERS-CoV). Transmitted from an animal reservoir in camels, MERS was identified in September 2012 and continues to cause sporadic and localized outbreaks. The third novel coronavirus to emerge in this century is called SARS-CoV-2. It caused coronavirus disease 2019 (COVID-19), which emerged from China in December 2019 and was declared a global pandemic by the World Health Organization on March 11, 2020 (6, 7).

Corresponding author: Samanwita Banerjee, Ayurvet Research Foundation, Chidana, Haryana, India. E-mail: samanwita.banerjee@arfmail.in.

\section{Morphology}

The name "coronavirus" is derived from Latin corona, meaning "crown" or "wreath due to the characteristic appearance of virions, which have a fringe of large, bulbous surface projections creating an image reminiscent of a crown or of a solar corona. It is spherical and pleomorphic and having a diameter of 0.12 micrometer. The virus envelops consist of lipid bi layer with structural protein such as membrane, spike anchored on it. There are small spike like surface proteins also known as hemagglutanin esterase (HE). Inside the envelope, there is the nucleocapsid, which is formed from multiple copies of the nucleocapsid $(\mathrm{N})$ protein, which are bound to the positive-sense single-stranded RNA genome in a continuous beads-on-a-string type conformation. The combined structure protects the virus outside the host cell as shown in Fig 1. The genome one of the largest among RNA viruses which size ranges from 26.4 to 31.7 kilobases and has a $5^{\prime}$ methylated cap and a $3^{\prime}$ polyadenylated tail (3). The twothirds of the genome ORF1a/b encodes polyproteins, which form the viral replicase transcriptase complex and the other ORFs on the one-third of the genome encode four main structural proteins: spike (S), envelope (E), nucleocapsid $(\mathrm{N})$ and membrane (M) proteins, as well as several accessory proteins (8).

\section{Modes of transmission}

Patients with COVID-19 show symptoms such as fever, nonproductive cough, sneezing, dyspnea, myalgia, fatigue, normal or decreased leukocyte counts, shortness of breathing and radiographic evidence of pneumonia which are similar to 
the symptoms of SARS-CoV and MERS-CoV infections (8). People get infected in the neighbourhood of infected person who has symptoms from the virus includes cough and sneezing as it spreads through airborne zoonotic droplets shown in Fig. $2(4,6)$. The severity and onset of COVID-19 is dependent on age and immunity of the patients. The incubation period ranges from 2- 14 days and can be last for more than 2 weeks $(2,10)$. Many studies showed that Patients with comorbidity such as hypertension, coronary heart disease, obesity, are at high risk $(10,11)$.

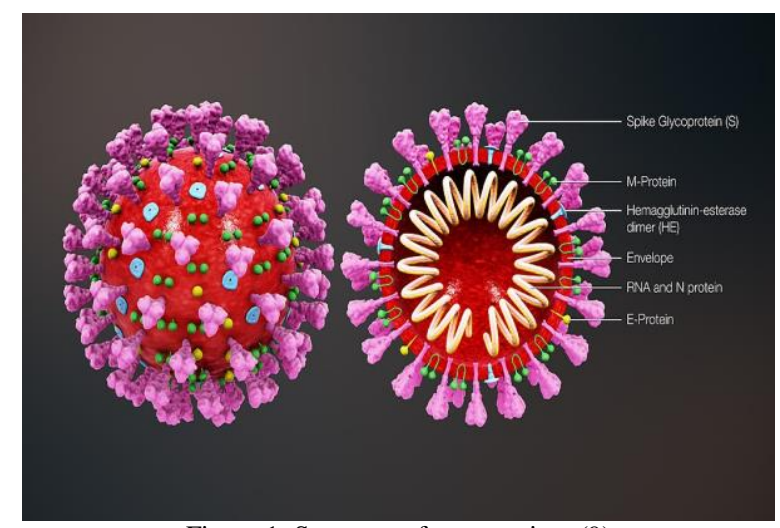

Figure 1: Structure of corona virus (9)

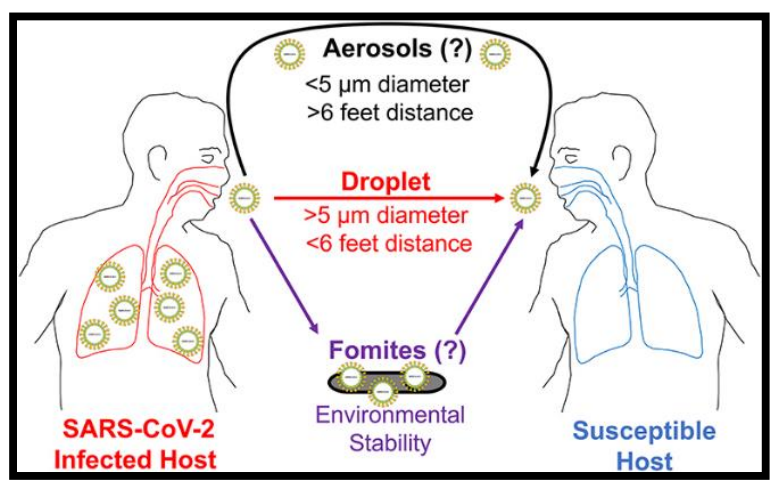

Figure 2: Airborne zoonotic droplets transmission (12)

\section{Pathogenesis of COVID 19}

The pathogenic mechanism of Coronavirus seems to be complex (10). The life cycle of the corona virus includes four steps i.e. attachment and entry, Replicase protein expression, replication and transcription and assembly and release (1). The virus enters into the host body through the mouth, nose and eye. From there, the virus makes its way down into the air sacs inside your lungs, known as alveoli. Once in the alveoli, the envelope spike glycoprotein present in the virus binds to its cellular receptor, angiotensin-converting enzyme 2 (ACE2) for SARS-CoV-2. Once the virus' RNA has entered a cell, new copies are made and the host cell is killed in the process, releasing new viruses to infect neighboring cells in the alveolus and cause inflammation in lung which in turn activate the immune response. During this process of unfolds, fluid begins to accumulate in the alveoli which stimulate nasal secretion, dry cough, sneezing and making breathing difficult. In severe cases, the immune system's response to the inflammation cause cytokine storm i.e. it starts affecting its own body cells than to virus it's trying to defeat which lead to acute respiratory distress syndrome (ARDS). At this stage, the surfactant that helps keeping the alveoli being collapsing has been diluted and fluid containing debris is imparing the gas exchange process that supplies oxygen to our bloodstream. In severe cases, it enters into the bloodstream, resulting in septic shock and multiple organ failure $(10,13,14)$. To better survive in host cells, SARS-CoV and MERS-CoV use multiple strategies to avoid immune responses (8).

\section{Diagnosis of COVID 19}

Clinical diagnosis of COVID-19 is mainly based on epidemiological history, clinical manifestations and some auxiliary examinations, such as nucleic acid detection, CT scan, immune identification technology (Point-of-care Testing (POCT) of IgM/IgG, enzyme-linked immunosorbent assay (ELISA) and blood culture. However, the clinical symptoms and signs of patients infected withSARS-CoV-2 are highly atypical, including respiratory symptoms, cough, fever, dyspnea, and viral pneumonia. Therefore, auxiliary examinations are necessary for the diagnosis of COVID-19, just as the epidemiological history. There are two commonly used nucleic acid detection technologies for SARS-CoV-2 are real-time quantitative polymerase chain reaction (RT-qPCR) and high-throughput sequencing (8). The high throughput sequencing is limited due to its equipment dependency and highly expensive whereas RT-q PCR is effective and commonly used for the detection of viruses from the respiratory secretion of patients. In this RT q PCR techniques two primers and probes are used in ORF1ab and $\mathrm{N}$ gene region for the detection. RT qPCR has to detect both the targeted region of viral genome present in the patients to be confirmed as infected. Although RT qPCR are specific but false negative rate cannot be denied (8). So, some of the medical practitioner use Ct scan of the chest is along with the RT q PCR. In CT Scan of the patients shows clear view of the abnormalities if presents. The typical CT images show bilateral pulmonary parenchymal ground-glass and consolidative pulmonary opacities, sometimes with a rounded morphology and a peripheral lung distribution $(8,10)$. Both the diagnosis method has its own limitation. Currently, POCT of IgM/IgG and ELISA kits for SARS-CoV-2 have been developed and pre-tested by some companies and have shown higher detection rates than nucleic acid detection $(8,15)$. Another laboratory investigation that are based on the count of white blood cell count. The lymphocyte counts less than 1000 has been associated with severe disease. There also decrease level of platelet count (15). It is necessary for the development of specific and detection kit for the diagnosis of COVID-19.

\section{Prevention and its mechanism}

In world, the cases of COVID 19 increases with high rate, so it is very much essential to opt the prevention. COVID 19 is inversely proportional to the immune system of the individual. Most of the people get infected with COVID-19 have mild symptoms and recover due to the proper medication, early stage or good immunity, whereas some patients are deceased due to the low immunity with having the previous diseases such as asthma, heart disease, diabetics, etc. there is no clinically proven drug for the treatment of COVID-19. Prevention is the only way to stay safe and healthy. As per the WHO guidelines, there are few prevention guidelines which need to be followed during this pandemic.

1. Washing of Hand Frequently: Washing your hands with soap and water or using alcohol-based hand rub regularly and thoroughly kills viruses that may be on your hands $(16,17)$. 
2. Maintain social distancing: Maintain at least 1 metre (3 feet) distance between yourself and anyone who is coughing or sneezing. When someone coughs or sneezes, they spray small liquid droplets from their nose or mouth which may contain virus. If you are too close, you can breathe in the droplets, including the COVID-19 virus if the person coughing has the disease $(16,17)$. Avoid touching eyes, nose and mouth: Hands touch many surfaces and can pick up viruses. Once contaminated, hands can transfer the virus to your eyes, nose or mouth. From there, the virus can enter your body and can make you sick $(16,17)$.

3. Practice respiratory hygiene: Make sure you, and the people around you, follow good respiratory hygiene. This means covering your mouth and nose with your bent elbow or tissue when you cough or sneeze. Then dispose of the used tissue immediately. Droplets spread virus. By following good respiratory hygiene, you protect the people around you from viruses such as cold, flu and COVID-19 (16, 17).

4. Seek medical care early: Stay home if you feel unwell. If you have a fever, cough and difficulty breathing, seek medical attention and call in advance (16).

5. Cloth face covering: Wear cloth face covering in the public setting where the social distance cannot be opted. It is additionally advised to use cloth mask rather than N95 mask to avoid spreading of the virus and help people who may have the virus and do not know it from transmitting it to others (18).

6. Disinfecting surfaces: Proper cleaning of frequently touched surfaces such as door, cabinet, etc. has to be performed using household detergent and water because COVID-19 virus survives on surfaces for many hours (19).

Apart from the aforementioned steps, there are several other steps, which can be opt by individual as a prevention such as resting and avoiding overexertion, drinking sufficient amount of water, avoiding smoking and smoky areas, etc. (1).

\section{Current development strategies on treatment of COVID 19}

Currently, there is no clinically approved drug or vaccines are available which show effectiveness against COVID-19. Many trials are on process for drug development in worldwide. The supportive treatments are under trial to relive discomfort during illness. In February Hydroxychloroquine was found to effectively reduce COVID-19 illness. Preliminary results from a trial indicated that chloroquine is effective and safe in COVID-19 pneumonia, "improving lung imaging findings, promoting a virus-negative conversion, and shortening the disease course but it has numerous, potentially serious, side effects. High doses of hydroxychloroquine are proving to be lethal (20). Remdesevir, adenosine nucleotide analog act as a viral RNA- dependent RNA polymerase inhibitor that inhibits RNA synthesis in coronavirus and act as an antiviral agent (21). Recombinant human monoclonal antibody can bind with receptor binding site and can be effective against COVID 19 infection. Vaccine development with a live-attenuated virus, viral vectors, inactivated virus, subunit vaccines, recombinant DNA, and proteins vaccine is another mode of strategy which can reduce the severity of outbreak (8). Serum Institute if India, Moderna BioNtech USA and others are in various stages of vaccine development. It will take at least 18 months or by late 2021 to establish effectiveness and safety.

\section{Using existing therapies and repurposing drugs to save lives}

With no sure shot vaccine/ drug in sight till late 2021, medical practioners are using existing therapies and repurposing drugs to save lives.

a. Combination of Hydroxychloroquine and the antibiotic azithromycin has shown some effectiveness in a very small French study and reports from China. Chloroquine act as an effective antiviral drug against SARS-CoV-2 infection at entry and post entry its stage and prevent the virus cell fusion by interfering with glycosylation of ACE2 receptor and its binding with spike protein $(21,22)$.

b. Arbidol which is also known as Umifenovir inhibits viral fusion in the targeted membrane, that blocks the viral entry and Oseltamivir suppresses the viral neuraminidase and inhibits the release of viral particle into the targeted cells $(22,23)$.

c. India has so far approved the combination of two antivirals drugs (Lopinavir and ritonavir) that targets viral protease to treat COVID -19 (20).

d. Convalescent plasma (Antibody therapy) - Giving sick patients with antibody rich plasma from those who have recovered is a century old therapy with proven track records.

e. Clot bursting drugs - tissue plasminogen activator is used to treat heart attack and stroke patients, may work to treat Covid-19. Clinical trials will take few months before safety is ensured.

f. Ayurvedic medicines and herbal drugs are also used along with the therapeutic drug for boosting the immunity. Specific prophylaxis includes Sanjeevani bati, chitrakadi, combination of Guduchi, (Tinospora cordifolia) Shunthi (Zingiber officinale) and Haridra (Curcuma longa Linn.). Sanjeevani bati act against cold cough and communicable diseases and also strengthens and rejuvenates the immune system. Other herbs such, Ocimum sanctum, Glycyrrhiza glabra, Adhatoda vasica, Andrographis paniculata, Swertiachirata, Moringa oleifera, Triphala and Trikatu has antiviral properties and inhibits the viral protease (24).

\section{Conclusion}

The sudden outbreak of the infectious disease COVID-19 is a threat to each and every individual globally. The remarkable point of this infection is its spreading rate among the communities. Scientific communities are working on the development of drugs and vaccines to eradicate the disease from the root level. During the period of this pandemic, it is necessary to opt the precautionary measures and avoid the spreading among the community. It is also important not to believe the rumors about the pandemic and depend on the reliable and authentic information. Immunity plays an important role in this disease, so it important have nutritious and healthy food to boost the immunity as per ministry of AYUSH, Govt. of India.

\section{Aknowledgment}

We would like to convey our thanks to Shri Mohan Ji Saxena, Managing Trustee for his valuable guidance and support. 


\section{Ethical issue}

Authors are aware of, and comply with, best practice in publication ethics specifically with regard to authorship (avoidance of guest authorship), dual submission, manipulation of figures, competing interests and compliance with policies on research ethics. Authors adhere to publication requirements that submitted work is original and has not been published elsewhere in any language.

\section{Competing interests}

The authors declare that there is no conflict of interest that would prejudice the impartiality of this scientific work.

\section{Authors' contribution}

All authors of this study have a complete contribution for data collection, data analyses and manuscript writing.

\section{Reference}

1. Shrikrushna Subhash Unhale, Quazi Bilal Ansar, Shubham Sanap , Suraj Thakhre, Shreya Wadatkar, Rohit Bairagi, Prof. Suraj Sagrule and Prof. Dr. K. R. Biyani, A REVIEW ON CORONA VIRUS (COVID-19), World Journal of Pharmaceutical and life sciences, 2020, Vol. 6, Issue 4, 109-115.

2. Harapan Harapan, Naoya Itoh, Amanda Yufika, Wira Winardi, Synat Keam, Heyhpeng Te, Dewi Megawati9,10, Zinatul Hayati1, Abram L. Wagner, Mudatsir Mudatsir, Coronavirus disease 2019 (COVID-19): A literature review, Journal of Infection and Public Health, 2020,https://doi.org/doi:10.1016/j.jiph.2020.03.019

3. Coronavirus,https://en.wikipedia.org/wiki/Coronavirus.

4. Syed Adeel Hassan, Fahad N. Sheikh, Somia Jamal, Jude K. Ezeh, Ali Akhtar, Coronavirus (COVID-19): A Review of Clinical Features, Diagnosis, and Treatment, Cureus, 2020, 12(3): e7355.

5. Anthony R. Fehr and Stanley Perlman, Coronaviruses: An Overview of Their Replication and Pathogenesis,), Coronaviruses: Methods and Protocols, Methods in Molecular Biology, 2015, vol. 1282, DOI 10.1007/978-1-4939-2438-7_1.

6. Dharmendra Kumar, Rishabha Malviya, Pramod Kumar Sharma, Corona Virus: A Review of COVID-19, Eurasian Journal of Medicine and Oncology, 2020;4(1):8-25.

7. Coronaviruses,https://www.niaid.nih.gov/diseasesconditions/coronaviruses , National institute of allergy and infectious diseases.

8. Xiaowei Li, Manman Geng, Yizhao Peng Liesu Meng, Shemin $\mathrm{Lu}$, Molecular immune pathogenesis and diagnosis of COVID-19, Journal of Pharmaceutical Analysis, (2020), Volume 10, Issue 2, April 2020, Pages 102-108)

9. Image courtesy: Scientific Animations. http://www.scinews.com/medicine/common-humancoronaviruses-sharply seasonal-08309.html .

10. C Francesco Di Gennaro, Damiano Pizzol, Claudia Marotta, Mario Antunes, Vincenzo Racalbuto, Nicola Veronese and Lee Smith, Coronavirus Diseases (COVID-19) Current Status and Future Perspectives: A Narrative Review, International Journal of Environmental Research and Public Health, 2020, 17, 2690; doi:10.3390/ijerph1708269.

11. Fei Zhou, Ting Yu, Ronghui Du, Guohui Fan, Ying Liu, Zhibo Liu, Jie Xiang, Yeming Wang, Bin Song, Xiaoying Gu, Lulu Guan, Yuan Wei, Hui Li, Xudong Wu, Jiuyang Xu, Shengjin Tu, Yi Zhang, Hua Chen, Bin Cao, Clinical course and risk factors for mortality of adult inpatients with COVID-19 in Wuhan, China: a retrospective cohort study, The Lancet, 2020, Volume 395, Issue 10229, Pages 1054-1062.

12. Thushara Galbadage, Brent M. Peterson1 and Richard S. Gunasekera, Does COVID-19 Spread Through Droplets Alone, Frontiers in public health, (2020), https://doi.org/10.3389/fpubh.2020.00163.

13. Nick Routley, This visualisation shows what COVID-19 does to your body, https://www.weforum.org/agenda/2020/04/thisgraphic-shows-what-covid-19-does-to-your-body, World economic forum, (2020)
14. Peng Xie1, Wanyu Ma, Hongbo Tang and Daishun Liu, Severe COVID-19: A Review of Recent Progress With a Look Toward the Future, Frontiers in Public Health, (2020), https://doi.org/10.3389/fpubh.2020.00189.

15. Tanu Singhal, A Review of Coronavirus Disease-2019 (COVID19), The Indian Journal of Pediatrics, (April 2020) 87(4):281-286.

16. Coronavirus disease (COVID-19) advice for the public, WHO, https://www.who.int/emergencies/diseases/novel-coronavirus2019/advice-for-public .

17. Amirreza Talaiekhozani, A Short Communication on COVID-19 Outbreak, Journal of Infertility and Reproductive Biology, 2019, Volume 7, Issue 4, Pages: 27-28.

18. Recommendation Regarding the Use of Cloth Face Coverings, Especially in Areas of Significant Community-Based Transmission, Centre for disease control and prevention, https://www.cdc.gov/coronavirus/2019-ncov/prevent-gettingsick/cloth-face-cover.html

19. COVID 19, prevention tips, Web Med network, https://www.medicinenet.com/covid19 prevention tips/article.htm

20. COVID-19 drug development, https://en.wikipedia.org/wiki/COVID-19_drug_development

21. Suranga L Senanayake, Drug repurposing strategies for COVID19, Future drug discovery, 2020, vol. 2, no.2, https://doi.org/10.4155/fdd-2020-0010 .

22. Renyi Wu \& Lujing Wang \& Hsiao-Chen Dina Kuo \& Ahmad Shannar \& Rebecca Peter \& Pochung Jordan Chou \& Shanyi Li \& Rasika Hudlikar \& Xia Liu1, \& Zhigang Liu1 \& George J. Poiani \& Louis Amorosa \& Luigi Brunetti \& Ah-Ng Kong, An Update on Current Therapeutic Drugs Treating COVID-19, Current Pharmacology Reports, 2020, https://doi.org/10.1007/s40495020-00216-7

23. Sandro G. Viveiros Rosa1 and Wilson C. Santos, Clinical trials on drug repositioning for COVID-19 treatment, Pan American Journal of Public Health, 2020, https://doi.org/10.26633/RPSP.2020.40

24. Sanjeev Rastogi, Deep Narayan Pandey, Ram Harsh Singh, COVID-19 pandemic: A pragmatic plan for ayurveda intervention, Journal of Ayurveda and Integrative Medicine, 2020, doi.org/10.1016/j.jaim.2020.04.002. 